\title{
Managing the Real-time Behaviour of a Particle Beam Factory: The CERN Proton Synchrotron Complex and its Timing System Principles.
}

\author{
J. C. Bau, G. Daems, J. Lewis, J. Philippe \\ PS Division, CERN, CH-1211 Geneva 23, Switzerland
}

\begin{abstract}
In the CERN 26 Gev Proton Synchrotron (PS) accelerator network, super-cycles are defined as sequences of different kinds of beams produced repetitively [Fig.1]. Each of these beams is characterised by attributes such as particle type, beam energy, its route through the accelerator network, and the final end user. The super-cycle is programmed by means of an editor through which the operational requirements of the physics programme can be described. Each beam in the normal sequence may later be replaced by a set of spare beams automatically depending on software and hardware interlocks and requests presented to the Master Timing Generator (MTG [Glos. 1]). The MTG calculates at run time how each beam is to be manufactured, and sends a telegram [Glos. 3] message to each accelerator, just before each cycle, describing what it should be doing now and during the next cycle. These messages, together with key machine timing events and clocks are encoded onto a timing distribution drop net where they are distributed around the PS complex to VME-standard timing reception TG8 [Glos. 8] modules which generate output pulses and VME bus interrupts for task synchronisation. The TG8 modules are able to use accelerator-related clocks such as the incremental/ decremental magnetic field trains, or the beam revolution and radio frequencies to produce high precision beam synchronous timing. Timing Surveillance Modules (TSM) monitor these timings, which give high precision interval measurements used for the machine tuning, beam diagnostics, and fault detection systems.
\end{abstract}

\section{INTRODUCTION}

CERN is unique in possessing the largest network of interconnected particle accelerators in the world. Getting the best out of this network, among other things, requires highly coordinated timing systems, and strategies for accommodating dynamic operational requests. With the coming of the new machines Anti-proton Decelerator, (AD) and the Large Hadron Collider (LHC), the need for a centralised and uniform CERN wide timing strategy is becoming very apparent, because full exploitation of these new machines requires very dynamic response capabilities from their injector chains. It is towards this aim that collaborations have been established between the two CERN accelerator divisions (PS [Glos. 4] and SL), which have given rise to a common timing hardware standard based on the MTG and TG8 [Glos. 1, 2] modules. In the PS division, there have been as many as nine linked particle accelerators working together in a coordinated network based on the PulseTo-Pulse (PPM [Glos. 6]) [1] control mechanism, and currently employing the new hardware standard MTG/TG8.

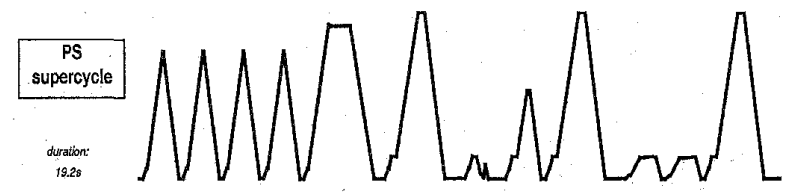

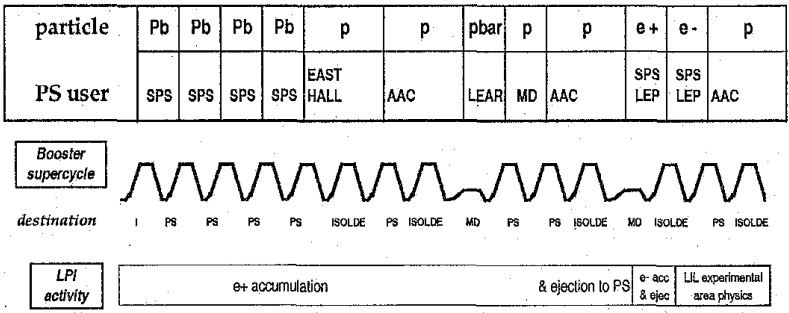

Figure1: Example of PS super cycle used in fall 96.

This paper describes the new PS timing system which became fully operational in March 1997, and discusses how we see it evolving under the $\mathrm{AD}$ and $\mathrm{LHC}$ requirements.

\section{GENERATING THE TIMING}

For each beam to be produced by the PS beam factory, the corresponding fixed flight paths through the accelerator network, and the execution time of each cycle, can be used to deduce a strategy for manufacturing them, based on some optimisation criteria. What results from this process are time ordered sets of cycles for each accelerator in the network, which are executed concurrently so that the beams are transferred from one accelerator to the next until ejected from the network. This process is governed by an interactive beam sequence editor programme, which builds the sequence strategy to be sent to the MTG for execution [2]. The MTG accepts the sequence, and builds telegram messages which describe the present and next cycles of the accelerators, and sends them out just before the cycle is about to execute [Fig.2]. The editor builds not just one beam sequence, it also includes an alternative sequence, which permits the MTG to dynamically switch between them at run time, based on request and interlock conditions. This switching in of a spare beam must be coordinated across all the accelerators in both the normal and spare beams so that beam integrity is respected.

In addition to the telegrams, the MTG also calculates the coarse timing [Glos. 8] events needed to process the beam for each cycle execution. Each cycle can be broken into an 
injection, acceleration, and ejection phase. The timing for each ejection phase is the slave of the injection of the next accelerator along the beam path.

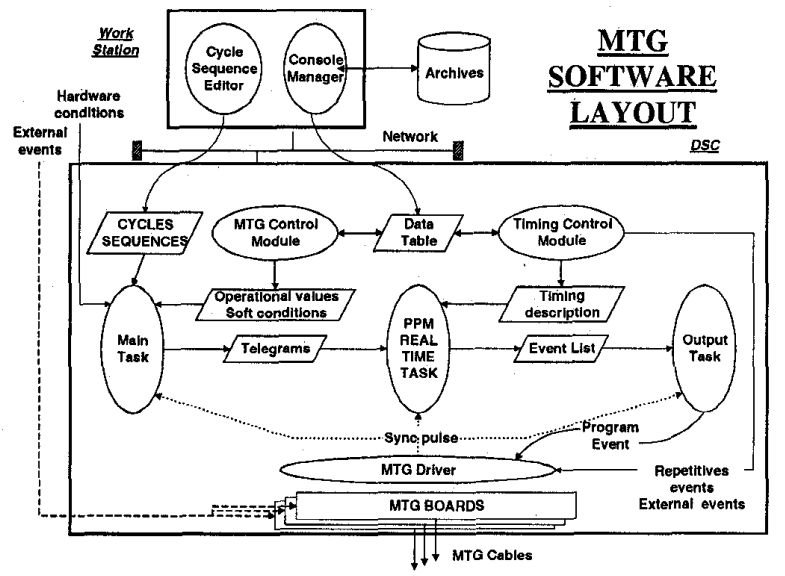

Figure 2: MTG Software Layout

The final ejection from the network is its own master. The MTG is able to calculate these timings from the telegram itself containing the beam destination, and a read only copy of the timing descriptions from the central database. The functionality required is provided by the standard PPM mechanism, by disabling ejection timing if the beam destination is another machine and enabling it if the beam destination is an end user.

The timing description used by the MTG to generate the coarse timing [Glos. 8] is organised into two classes, Virtual Master (VM) timings, and Slave Key (SK) timings. A VM timings is purely conceptual, it exists only to define a reference instant from which a family of SK timings can be specified in positive (post pulse) or negative (pre pulse) time with respect to it. It is the VM event, which can be set specifically for each cycle according to the PPM rules. If the VM event is disabled, then none of the pulses in the SK family will be produced.

\section{THE MTG HARDWARE}

The current MTG contains several VME-standard timing generation modules developed at CERN. Each module drives one timing drop net, and produces serially encoded events with a time resolution of $1 \mathrm{~ms}$ and a precision of $0.25 \mu \mathrm{s}$. Up to 5 events can be sent per ms, which can contain the GPS date and time, telegram events, key timing events, and a $1 \mathrm{KHz}$ clock.

A stable $10 \mathrm{MHz}$ Rubidium gas oscillator is divided down to a $1 \mathrm{KHz}$ train and a $1.2 \mathrm{~s}$ train, which are fed in parallel to the MTG modules, which in turn provide a $1.2 \mathrm{~s}$ bus interrupt to the VME host processor. Each interrupt, the host prosessor calculates the events for the current time slice, builds an event table for the MTG modules, which broadcast the events over the timing drop nets. Some external events, such as those associated with a measurement of the magnetic field, which can not be calculated by the MTG, are input via the P2 connector for redistribution over the drop net.

A standby-slave MTG can take over from the master in the case of failure, and more importantly, during routine maintenance [Fig.3]. The switch over is activated manually, the new master is requested by the switch to take control, it copies to memory all the latest settings from disc, and then informs the switch that it is ready to take master-ship, the switch then routes its outputs to the control system. There can be no brutal shift in super-cycle phase at switch over, so the master and slave MTG systems must be beam synchronised.

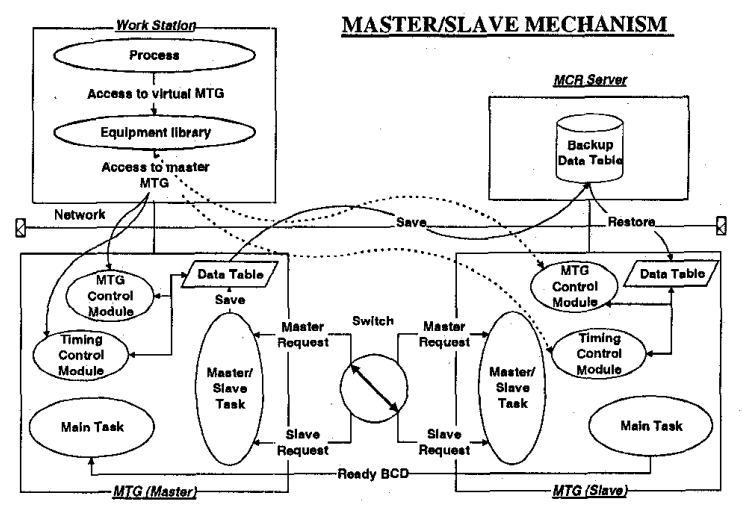

Figure 3: Master/Slave Mechanism

\section{TIMING RECEPTION}

The timing is distributed to some 250 TG8 modules [3] located around the PS complex which decode the events on the timing drop net, and determine for each, whether or not some action should be carried out. The permitted action can be either programming one of the eight on board counters to produce an output pulse, or provoking a VME bus interrupt, or both. An important feature of the TG8 firmware is the ability to trigger an action on the combination of a key timing event and a telegram condition such as the particle type, or beam destination. So once the module has been programmed, it will continue to function without any VME host intervention outputting the timing autonomously according to the telegram. The PPM mechanism is thus entirely supported by the TG8 firmware directly [Fig.4].

The TG8 is provided with external clock and start inputs, and hence all the usual tricks timing specialists do with counters are available. However, a counter is loaded only by an action triggered from the incoming event stream, and hence, it can not be used as a divider.

\section{TIMING SurveILlanCE}

The TG8 module uses all counter outputs to provoke local $\mathrm{CPU}$ interrupts, which enables it to create a history 


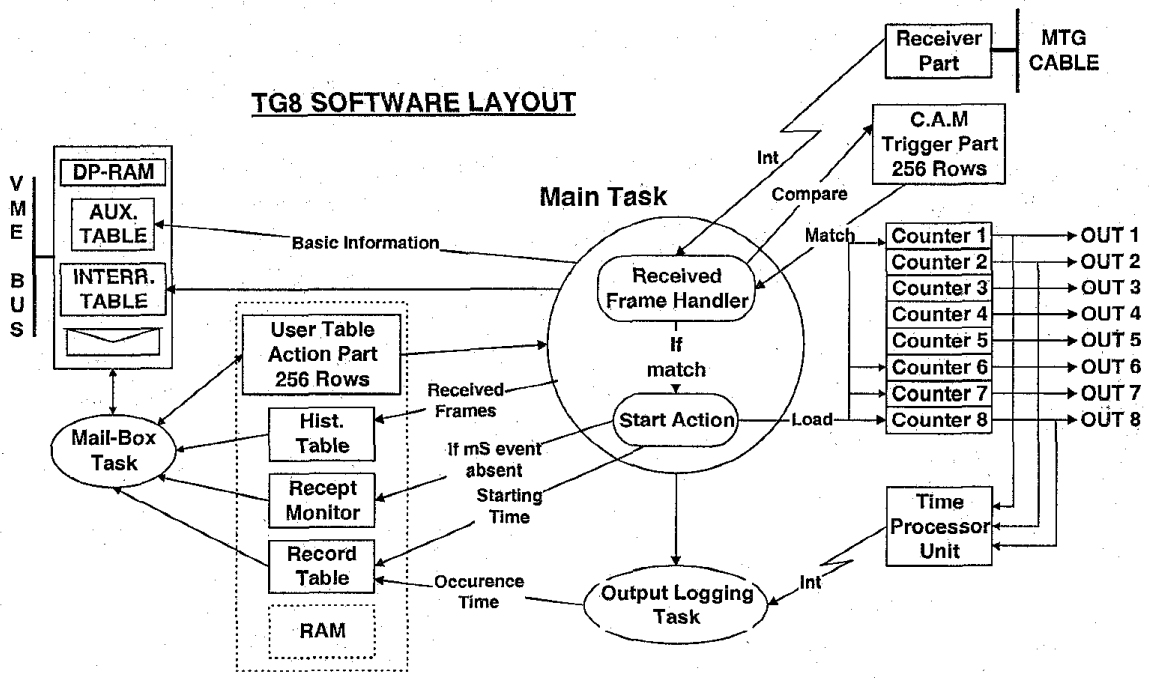

Figure 4: TG8 Software Layout

mechanism, in which all output pulses are logged with a time stamp derived from the GPS-time [Glos. 7] event and the $1 \mathrm{KHz}$ clock, giving a time resolution of $1 \mathrm{~ms}$. This proves to be a useful built in diagnostic tool, providing not only a log of all output pulses, but also of bus interrupts, and telegram arrival times. The coarse timing events themselves are derived from the same $1 \mathrm{KHz}$ clock, and so in this case the TG8 history mechanism provides an adequate monitor. In particular, one TG8 for each timing generation module in the master and slave MTG systems is used to monitor that modules timing events. A surveillance task compares the timings calculated by the VME host, with what actually arrives at the TG8 modules, and hence the complete event generation chain is monitored. For the more precise timing surveillance required by the fine timing, Timing Surveillance Modules [4]. (TSM [Glos. 5]) are installed near the TG8 modules. The stable $10 \mathrm{MHz}$ clock distributed around the PS complex, is used by TSM modules to measure the time between an arbitrary reference pulse, and up to 31 other timing input channels coming from the TG8 outputs. The advantage of using a common reference clock for all the TSM modules, is that inter module clock drift is eliminated, allowing timing comparisons to be made directly. The clocks driving the MTG systems are derived from dividing down the same reference clock, so there is no drift between the measurement system, and that that generates the timing. This approach to timing surveillance has greatly simplified the analysis of the TSM data. Because the TSM modules receive the TG8 outputs, they are further able to monitor the same electrical signal that is received by the client, and hence more of the timing distribution chain is checked.

The timing surveillance system serves two very different clients, the accelerator operations team, and the controls specialists. On the operations side, it is usually enough to provide the acquisition value, while the controls specialists are more interested in verifying that the timing system is functioning correctly, that is according to its description in the data base. This has prompted development of a significant number of diagnostic tools displaying the timing layout, acquisition and control values on the PC platforms, to evolve in parallel with main stream standard model UNIX based control system.

\section{FutURE EXTENSIONS FOR THE ANTIPROTON DECELERATOR (AD) AND THE LARGE HADRON COLLIDER (LHC)}

Until now, each beam produced by the PS complex has had a known flight path through the accelerator network, enabling us to predetermine schedules to produce the required sets of beams for the physics programmes in advance. These schedules are tightly coupled fixed length super-cycles with spare and normal sequences chosen on the bases of external requests and interlocks. By June 1998 the PS-MTG will produce the $\mathrm{AD}$ timing, which must provide a loosely coupled capability, in which the $\mathrm{AD}$ machine [5] has a single independent variable length long cycle, but which rendezvous occasionally with the PS machine for beam transfer. The AD cycle can last from minutes to hours and may receive several filling batches from the PS, which it will then cool. The AD operations team may decide the time spent cooling, and the number of batches, while the cycle is executing. To solve this problem, the $\mathrm{AD}$ cycle is split into segments, and the cycle is built up from a list of these segments in the same way as we build super-cycles up from cycles. The difference between segments and cycles is that between segments the $\mathrm{AD}$ cycle can be stopped permitting waiting on any plateaux as long as needed, or a set of segments can be repeated permitting multiple batch filling. For this type of machine, it is impossible to specify fixed beam schedules in advance, thus beam transfers to the $\mathrm{AD}$ machine must be negotiated with the PS injector chain as needed in real time. One way to achieve this, 
without wasting injector cycles, is to change the injector chain super-cycles dynamically to deliver the requested $\mathrm{AD}$ beam batches, after which the injectors are free to perform other tasks until they are needed again. We call this type of negotiation mechanism loose coupling because the phase and position relationship between the injector cycles is only fixed at run time during beam production. Starting at the first injector and following the beam down line direction, if all down line machines are ready to receive the beam, it is delivered, otherwise the injectors can execute cycles of another beam. Looking at the down line machines, if they require beam, they must wait for their injectors to deliver it. Thus each down line machines resynchronises on the first available cycle of its injector to determine the correct phases between the cycles for beam transfer in real time. Notice that now the super-cycles of the machines no longer need to be the same length, and can be updated independently of each other. This loose coupling mechanism is thus recursive and applies to each injector sub chain, in which the last machine in the chain must resynchronise by waiting for the correct phase to receive the beam.

Today an independent SPS MTG operates with a fixed super-cycle which is triggered from the PS start-super cycle event, the PS then delivers requested beams in fixed cycle positions according to a prearranged agreed sequence, the machines are strongly coupled. In 1979 the SPS timing system was modified for Pulsing/Coasting mode, when it was operated as a beam collider. In this mode the SPS timing could be suspended hence freezing the cycle at the desired energy for Proton/Antiproton collider operation. Filling was achieved in the usual way based on the PS start super-cycle event and delivery of an agreed sequence of beams according to the preprogrammed SPS MTG super-cycle. The two independent timing systems each have to be set up according to an agreed schedule and the PS super-cycle for SPS has to be established prior to actual beam production. This dual MTG mechanism is now under review because in its present form, it is not sufficiently adaptable to meet the frequent LHC filling requests, which require dynamic changes in super-cycle length and composition, while simultaneously maximising beam production for other users such as Neutrino physics. The LHC is loosely coupled to its injector chain, which now includes both the PS and SPS machines, and hence for efficient usage of the LHC, and also all the machines in its injector chain, the timing should be centrally managed. Thus in the LHC era we would hope to see one central timing system coordinating and sequencing all the machines together as a single resource.

\section{ACKNOWLEDGMENTS}

We would like to thank Gary Beetham of CERN/SL Division for the fruitful collaboration during the design of the original concepts, Bruno Puccio of CERN/SL Division for the hardware design, testing and implementation of the MTG and TG8 VME modules, and Vladimir Kovaltsov of IHEP/Protvino-Russia for the firmware and drivers for the MTG and TG8 VME modules and the sequence editor.

\section{REFERENCES}

[1] J. Boillot, G. Daems, M. Overington, P. Heymans, "Pulse to Pulse Modulation of the Beam Characteristics for the Beam Characteristics and Utilisation in the CERN PS Accelerator Complex", IEEE Trans. on Nucl. Science, Vol. NS-28, nr 3, p. 2191 (1981).

[2] J. Lewis, V. Sikolenko, "Providing the New CERN PS Timing System", Proc. ICALEPCS'93, Berlin, Germany, October 18-23, 1993, Nucl. Instr. And Meth. A352 p.91 (1994).

[3] C.G. Beetham G. Daems, J. Lewis, B. Puccio, "A New VME Timing Module”, Proc. ICALEPCS'91, Tsukuba, Japan, 11-15 November 1991, p. 360.

[4] G. Daems, W. Heinze, "Specification for a Timing Surveillance VME Module (TSM)", CERN Int. Report PS/CO/Note 96-36, 1996.

[5] S. Maury (editor), "Design Study of the Antiproton Decelerator: AD", CERN Int. Report PS/AR/Note 96-34, 1996.

\section{Glossary}

[1] MTG : The Master Timing Generator is a collection of modules in a VME crate that drives the timing distribution drop net.

[2] TG8 : Timing Generation VME module with 8 counters which receives events from the timing distribution drop net.

[3] TELEGRAM : The telegram is a message sent by the MTG over the timing distribution drop net just before each machine cycle, which describes the beam.

[4] PS : The $26 \mathrm{Gev}$ Proton Synchrotron itself, or the CERN division responsible for running it.

[5] TSM : The Timing Survey VME Module is a device that measures time intervals between a start pulse and up to 31 other input pulses. It can use an external $10 \mathrm{MHz}$ input, as in the PS case, or an internal $20 \mathrm{MHz}$ clock.

[6] PPM : Pulse to Pulse Modulation is the method used by the PS control system front end equipment control modules to select control and acquisition values according to the telegram each machine cycle.

[7] GPS time : The MTG system contains a VME module capable of receiving the satellite GPS (Global Position System) time of day and date signals which it then broad casts over the timing distribution drop net.

[8] Coarse Timing : The coarse timing is produced by the MTG as an event stream sent over the timing distribution drop net each ms. It includes the $1 \mathrm{KHz}$ reference clock, and up to 5 other events that represent fundamental features of the machine cycles such as, start and end of cycle, injection and ejection, and the start of the magnetic field increase, etc. 